\title{
Sistema de otimização do balanço de massas para alimentação de alto-fornos: um estudo de caso
}

\section{Lidia de Paula Pessoa}

lidiappessoa@hotmail.com

José Maria do Carmo Bento Alves

jmalves@uai.com.br

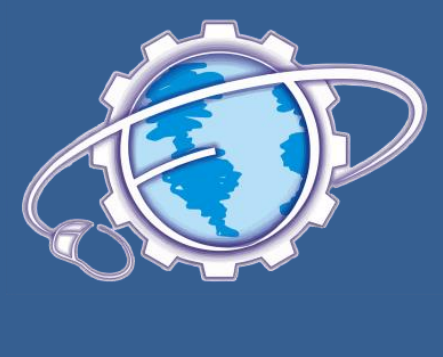

\section{RESUMO}

Este trabalho teve como objetivo apresentar um modelo matemático baseado em programação linear por metas para resolução do Problema do Balanço de Massas de um alto-forno. Tal problema consiste na determinação das quantidades de material que será enfornado. Sua solução deve ser obtida rapidamente, pois influencia diretamente na qualidade do ferro-gusa fabricado. Atualmente, a grande maioria das empresas utiliza o método da tentativa e erro baseando-se apenas na experiência dos funcionários para obter a solução do problema em questão. A metodologia empregada modelou e implementou o modelo proposto através do otimizador LINGO 10.0 em interface com planilhas do Microsoft Excel. Os resultados computacionais indicaram que tal modelo é capaz de gerar soluções ótimas rapidamente, possibilitando ao usuário analisar vários cenários antes da tomada de decisão.

Palavras-chave: Otimização, Programação matemática, Balanço de massas.

\section{Mass balance optimization system for blast furnace feed: a case study}

\begin{abstract}
The objective of this study is to present a mathematical model based on a targeted linear programming to solve the Problem of the Mass Balance in blast furnaces. Such a problem consists of determining the material quantity to furnace. Its solution must be quickly obtained since it directly influences the quality of the manufactured iron. Nowadays, the great majority of the industries use the trial-and-error method based only on the employees' experience to obtain a solution to the problem. The applied methodology modeled and implemented the proposed model though the LINGO 10.0 optimizer on interface with the Microsoft Excel spreadsheets. The computer results indicated that such a model is able to quickly generate excellent solutions, enabling the user to analyze several scenarios before making a decision.
\end{abstract}

Key words: Optimization; Mathematical Programming; Mass Balance. 


\section{Introdução}

Durante a Segunda Guerra Mundial, na Inglaterra, devido à necessidade da solução de problemas de grandes dimensões e complexidade, como de logística, estratégia militar e utilização dos recursos militares, grupos de cientistas deram início ao que seria chamado de Pesquisa Operacional. Com o objetivo de apoiar os comandos operacionais para a solução desses problemas, criaram-se grupos multidisciplinares de físicos, matemáticos, engenheiros e cientistas sociais para desenvolver modelos matemáticos a partir de dados e fatos com o intuito de simular e avaliar resultados hipotéticos de estratégias e decisões alternativas. Diante dos resultados bem-sucedidos da equipe inglesa, posteriormente os Estados Unidos foram motivados a desenvolver técnicas semelhantes.

Assim, apesar de a Inglaterra ter sido a precursora da Pesquisa Operacional, foi nos Estados Unidos que ela obteve maior propagação, com a equipe de cientistas liderada por George B. Dantzig. Tal equipe desenvolveu, durante a guerra, um método que recebeu o nome de Método Simplex, concluído em 1947.

Em razão do sucesso e fim da guerra, os cientistas resolveram aplicar a metodologia em problemas empresariais, alcançando bons resultados. Com a evolução da informática, criaram-se condições para desenvolvimento de softwares baseados em pesquisa operacional, permitindo sua aplicação em problemas de grande porte. Seguiram-se, então, grandes desenvolvimentos técnicos e metodológicos que hoje, com o apoio de meios computacionais de crescente capacidade e disseminação, permitem trabalhar enormes volumes de dados sobre as atividades das empresas e, através de adequados modelos de base quantitativa, simular e avaliar linhas de ação alternativas, bem como encontrar soluções que melhor servem aos objetivos dos indivíduos ou das organizações.

Em face do seu caráter multidisciplinar, a Pesquisa Operacional estende-se por praticamente todos os domínios da atividade humana, da Engenharia à Medicina, passando pela Economia e pela Gestão Empresarial.

Atualmente, a Pesquisa Operacional está sendo largamente utilizada nos diversos setores produtivos. E vem sendo vista como importante ferramenta para a tomada de decisão no meio empresarial, pois consegue simular diversos cenários otimizados com diferentes configurações quantitativas e qualitativas, cabendo aos responsáveis escolher a melhor opção para o momento

Uma característica importante que a Pesquisa Operacional possui e facilita muito o processo de análise de decisão é a utilização de modelos. Tais modelos permitem a "experimentação", o que significa que uma decisão pode ser mais bem avaliada e testada antes de ser efetivamente implementada. A economia de recursos e a experiência adquirida pela experimentação, por si só, justificam a utilização de técnicas de Pesquisa Operacional como instrumentos de administração de empresas.

Este trabalho apresenta também um estudo de caso feito em uma empresa siderúrgica de Minas Gerais, que aqui é denominada Empresa $A$. Atualmente, o método utilizado pela empresa é o da tentativa e erro, abrindo grande possibilidade para otimização do processo, visto que tal método se baseia somente no julgamento pessoal do funcionário responsável pela área.

Esta pesquisa teve como foco buscar uma aplicação da pesquisa operacional em uma empresa siderúrgica, utilizando a programação linear por metas para resolver o problema do balanço de massas do seu alto-forno, de modo que atenda aos limites de especificações de qualidade e quantidade preestabelecidas pelo cliente, satisfazendo as restrições operacionais.

\section{Referencial Teórico}

\subsubsection{Programação linear}

A Pesquisa Operacional divide-se em grandes áreas, entre as quais se encontra a Programação Linear. Esta técnica é utilizada para encontrar o máximo ou o mínimo de uma função, chamada de função objetivo, sujeita a algumas restrições. São ditos Problemas de Programação Linear porque a função objetivo e as restrições são equações e inequações lineares. Problemas desse tipo dizem respeito à atribuição e distribuição de recursos entre as diversas tarefas ou atividades que devem ser realizadas. O que se procura é a melhor distribuição possível dos recursos, entre as diversas tarefas ou atividades, de forma a atingir valor ótimo do objetivo estabelecido.

Dois passos são fundamentalmente necessários para resolução de um Problema de 
Programação Linear (PPL). O primeiro deles é a modelagem do problema e o segundo, a aplicação de uma técnica para resolvê-lo. O método mais utilizado é o Simplex. As equações de 1 a 3 indicam um Problema de Programação Linear na formapadrão. Bregalda et al. (1988) apresentaram a técnica para reduzir qualquer Problema de Programação Linear à forma-padrão.

$$
\operatorname{Min} \sum_{j=1}^{n} c_{j} x_{j}=Q(x)
$$

s.a.:

$$
\begin{aligned}
& \sum_{j=1}^{n} a_{i j} x_{j}=b_{i} \quad \text { onde } b_{i} \geq 0 \quad \forall i=1, \ldots, m \\
& x_{j} \geq 0 \quad \forall j=1, \ldots, n
\end{aligned}
$$

Nessa formulação $j=1, \ldots, n$ representa atividades a serem realizadas, $c_{j}$ o custo associado à $j$-ésima atividade, $x_{j}$ é uma variável de decisão que quantifica o nível de operação da $j$-ésima atividade, $i=1, \ldots, m$ representa restrições a serem atendidas, $b_{i}$ a quantidade de recursos disponíveis ou exigências a serem cumpridas e $a_{i j}$ é a quantidade de recurso $i$ (ou exigência) em uma unidade da atividade $j$. A equação 1 representa a função objetivo, que deve ser minimizada. Os conjuntos de equações 2 e 3 normalmente são denominados restrições do PPL, e o segundo denomina-se condição de não negatividade.

O método Simplex, fundamentado na Álgebra Linear, baseia-se na propriedade de que a solução ótima do PPL, se existir, está localizada em um vértice do politopo formado pelo conjunto das soluções viáveis do problema. Tal ponto é denominado Solução Básica Viável. Assim, o método consiste em gerar, a cada iteração, soluções básicas viáveis cada vez melhores. Quando não é mais possível melhorá-la, a solução atual é a solução ótima.

\subsubsection{Programação Linear por Metas}

A Programação Linear assume que as restrições não podem ser violadas e, geralmente, está condicionada à solução de um único objetivo. Entretanto, inúmeros problemas no mundo real exigem que boa parte das decisões do dia a dia das empresas seja flexível e estão relacionadas com objetivos diversos, que têm que ser atendidos simultaneamente. Assim, segundo Bueno e Oliveira (2004), a Programação Linear por Metas, ou Goal Programming, surge como técnica de pesquisa operacional que permite a modelagem e busca de soluções para problemas com múltiplos objetivos ou metas. Desse modo, segundo Costa (2005), pode-se caracterizar a programação linear por metas como uma extensão da programação linear que permite a resolução de problemas com múltiplas metas.

Uma das diferenças mais significativas da programação linear clássica para a programação por metas está na função objetivo, na qual é incorporado um componente de minimização dos desvios em relação às metas. Neste trabalho, utilizou-se a função de avaliação arquimediana, em que cada meta possui importância diferente na otimização e é hierarquizada através de pesos, que variam de valores baixos para metas irrelevantes até valores elevados para as metas mais importantes. Esta e outras metodologias de função de avaliação estão descritas em Romero (2004).

Outra característica da programação linear por metas é que determinadas restrições podem ser flexibilizadas com a adição de variáveis de desvio. Com tal estratégia, o espaço de soluções viáveis é ampliado, uma vez que as restrições flexibilizadas deixam de restringi-lo. Com as variáveis de desvio é possível, também, fazer uma análise de quanto uma restrição fica fora da meta original. Assim, incorporando a componente de minimização na função objetivo e aplicando a relaxação das restrições no modelo de programação linear, tem-se representado pelas equações de 4 a 7 o modelo genérico da programação linear por metas.

$$
\operatorname{Min} \sum_{j=1}^{n} c_{j} x_{j}+\sum\left(w_{i}^{+} d_{i}^{+}+w_{i}^{-} d_{i}^{-}\right)=Q(x)
$$

s.a.:

$$
\begin{array}{cc}
\sum_{j=1}^{n} a_{i j} x_{j}-d_{i}^{+}+d_{i}^{-}=b_{i} & \forall i=1, \ldots, m \\
x_{j} \geq 0 & \forall j=1, \ldots, n \\
d_{i}^{+}, d_{i}^{-} \geq 0 & \forall i=1, \ldots, m
\end{array}
$$

Neste PPL $, j=1, \ldots, n$ representa atividades a serem realizadas, $i=1, \ldots, m$ as restrições a serem atendidas, $b_{i}$ é a meta de recurso a ser utilizado ou exigência a ser atendida na $i$-éssima restrição, $c_{j}$ é o custo associado à $j$-ésima atividade, $x_{j}$ é a variável de decisão do problema que quantifica o nível de 
operação da $j$-ésima atividade e $a_{i j}$ é a quantidade de recurso $i$ (ou exigência) em uma unidade da atividade $j$. Além de tais variáveis originais da formulação do problema de programação linear anterior, são acrescentadas as variáveis de decisão $d_{i}^{+}$e $d_{i}^{-}$, que quantificam os desvios com relação às metas. A primeira, $d_{i}^{+}$, é chamada de Desvio Positivo da Meta $i$ e quantifica quanto a meta $b_{i}$ foi superada, enquanto a segunda, $d_{i}^{-}$, é chamada de Desvio Negativo da Meta $i$ e quantifica quanto falta para atingir a meta $b_{i}$. As constantes $w^{+}$e $w^{-}$ indicam, respectivamente, as prioridades dos $i$ ésimos desvios positivo e negativo em relação à meta. Resumindo, a equação 4 representa a função multiobjetivo. Os conjuntos de equações 5 a 7 representam as restrições do problema, sendo os conjuntos 6 e 7 denominados condições de não negatividade das variáveis de decisão.

\subsection{Siderurgia}

\subsubsection{Histórico}

O primeiro contato que o homem teve com o ferro foi por acaso, quando ele descobriu que algumas pedras (minério de ferro) utilizadas para rodear as fogueiras, onde madeiras eram queimadas, com o tempo tornavam-se maleáveis e mais resistentes. Ali acontecia a primeira redução do óxido de ferro pelo carbono da madeira. Com o passar dos anos, surgiu a necessidade de trabalhar maior quantidade de material. Para isso, foram utilizados fornos que mantinham a temperatura por mais tempo, chamados de baixos-fornos. Por volta do ano 1440 surgiu, na Alemanha, o primeiro altoforno, que tinha altura aproximada de 3 a $5 \mathrm{~m}$. Ele possuía capacidade de sopragem maior, tornando as temperaturas mais elevadas e facilitando, com isso, a incorporação do carbono ao ferro, o que diminui a temperatura de fusão. Com a utilização desse forno foi possível a produção do metal líquido, que foi chamado de ferro-gusa.

\subsubsection{Divisão do alto-forno}

As principais partes dos altos-fornos (Figura 2.1) são:

- Cadinho - possui forma cilíndrica com a função de coletar o ferro-gusa e a escória à medida que se formam. Seu resfriamento pode ser feito por meio de staves ou chuveiro externo à chaparia.

- Rampa - é a zona de fusão dos materiais, sendo na forma tronco-cônica. Pode ser revestida de refratário sílico-aluminoso ou grafita, e sua inclinação nos altos-fornos modernos está na ordem de $76^{\circ}$ a $82^{\circ}$ com a horizontal.

- Ventre - parte superior à rampa, tem forma cilíndrica, dependendo do perfil do alto-forno. Tal componente não é usado, ligando-se, direto, a rampa à cuba.

- Cuba - secção tronco-cônica com a base maior. É apoiada sobre o ventre ou o cadinho; sua função é compensar a dilatação dos componentes da carga durante a descida devido à escória e, consequentemente, impedir a criação de depósitos que se agarram nas paredes do alto-forno.

- Goela - é a parte por onde o alto-forno é abastecido. É revestida internamente por placas de desgaste que protegem o refratário do impacto da carga durante o enfornamento.

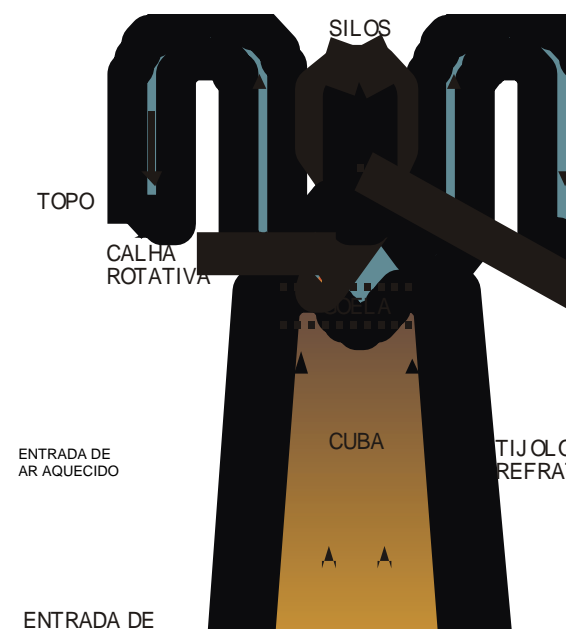

Figura 2.1 - Divisões do alto-forno

Fonte: FILHO; VIEIRA, 2007.

\subsubsection{Alimentação dos altos-fornos}

A alimentação do minério e do carvão nos altos-fornos é feita pela parte superior, de forma alternada e de modo que em seu interior formem camadas alternadas de minério e carvão, conforme pode ser visto na Figura 2.2.

Skip são os carros que fazem o carregamento de minério, fundentes e carvão no topo do altoforno. O carregamento do skip é feito por um distribuidor que monta a carga para que esta fique uniforme. 
Alguns altos-fornos operam com injeção de finos de carvão, o que feito por ventaneiras, que são sempre instaladas em pares. A injeção de finos causa redução na permeabilidade do leito, o que exigirá estudar o par custo $\times$ benefício, entre economizar carvão injetando os finos e baixar a produtividade do forno com a diminuição da permeabilidade do leito.

Os gases produzidos pelo alto-forno são enviados ao balão coletor; quando ele entra no balão, o diâmetro de escoamento do gás aumenta e, com isso, a velocidade do gás diminui. Por inércia e gravidade, o pó que é mais pesado continua o movimento e se deposita no fundo do balão. Já o gás quente sobe e sai pelas tubulações, que o levam para o lavador de gases.

Outro componente é o cowper, que tem a função de aquecer o ar que será injetado no altoforno, através da troca de calor feita com os tijolos refratários contidos no seu interior e que já se encontram aquecidos. Além dos cowpers, o aquecimento pode ser feito pelos glendons, que consistem de uma sala fechada fabricada de tijolos refratários e internamente possui uma tubulação de aço inox por onde passa o ar atmosférico que será aquecido e, posteriormente, injetado no alto-forno.

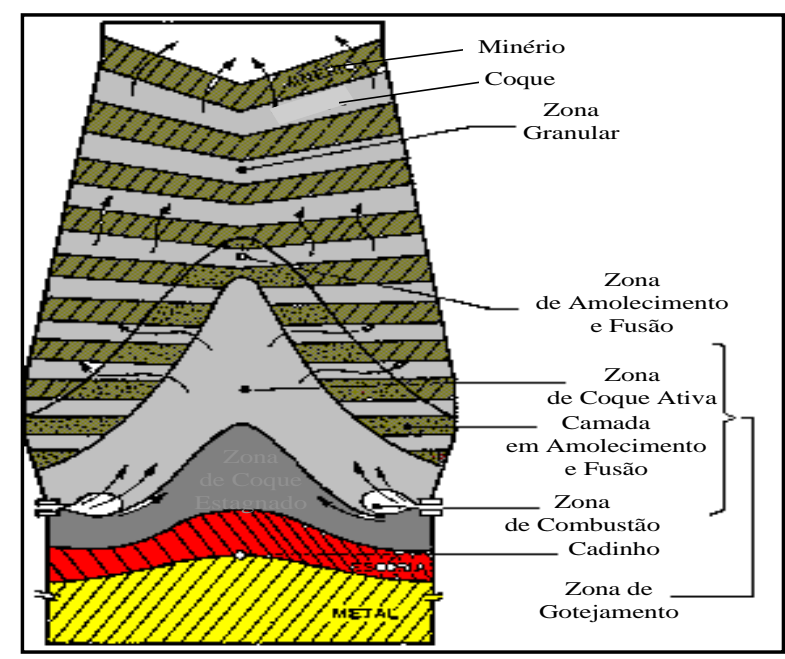

Figura 2.2 - Distribuição de cargas no alto-forno

Fonte: FILHO; VIEIRA, 2007.

\subsubsection{Siderurgia - Metalurgia do ferro}

A metalurgia do ferro consiste, basicamente, na redução de óxidos por meio de um redutor, o qual, em geral, é um combustível carbonoso. Os materiais carregados nos altos-fornos são: minério, combustível (redutor) e fundentes, que são utilizados para retirar as impurezas do minério e controlar a basicidade da escória, durante o processo de redução se transformam nos seguintes produtos: ferro-gusa, escória, gases de alto-forno e poeira. A redução dos óxidos de ferro se processa à medida que o minério, o agente redutor (coque ou carvão vegetal) e os fundentes (calcário ou dolomita) descem em contracorrente aos gases, provenientes da queima do carbono com o oxigênio do ar aquecido soprado pelas ventaneiras. Esse movimento ascendente dos gases e em sentido contrário ao do material é de suma importância para o trabalho do alto-forno.

$\mathrm{O}$ balanço de massas é feito de acordo com a necessidade do ferro-gusa, e este pode sofrer alterações de acordo com o minério, redutores e fundentes. Faz-se necessário explicar que a basicidade da escória é determinada em função da necessidade de maior ou menor dessulfuração do ferro-gusa e visa favorecer o seu escoamento, sendo, portanto, um dado de entrada fundamental para o balanço de massas.

O cálculo da basicidade pode ser feito de três formas: Binária, para análise da basicidade; Ternário, para análise da basicidade e fluidez; e Quaternária, para análise da viscosidade.

\section{Descrição do Problema}

Este trabalho enfoca uma siderúrgica integrada situada no interior do Estado de Minas Gerais, responsável pela produção de 980 t Ferrogusa/dia que escoa para a aciaria, transformando em $1.048,60 \mathrm{t}$ aço $/$ dia. O aço produzido é processado na laminação da unidade e enviado para outras unidades da empresa.

Para a produção do ferro-gusa, as entradas do alto-forno são os minérios, o carvão vegetal e os fundentes, que são levados ao topo do forno pelo skip, e as saídas são ferro-gusa, escória, gases e poeira. Os gases gerados na parte superior do altoforno passam por um processo de limpeza e, depois, são utilizados em diversos processos na empresa, como: aquecimento dos cowpers, aquecimento de panelas de ferro-gusa, caldeiras, reaquecimento dos tarugos, no processo de moagem de carvão, entre outros. A poeira e os gases inerentes do processo são seccionados por um sistema de captação. Durante $1 \mathrm{~h}$, o alto-forno fica tamponado para haver o gotejamento do ferro-gusa e da escória que são depositados no cadinho. Após esse tempo é feito um furo por onde o ferro-gusa vai correr, e somente $20 \mathrm{~min}$ após a escória começa a sair. Mesmo durante o tempo que o alto-forno está aberto, ele continua gotejando. $\mathrm{O}$ ferro-gusa corre até a panela, 
e a escória segue para o resfriamento por jato de água, passando por um processo de granulação, tornando-a matéria-prima para diversas empresas.

O carregamento é feito da seguinte maneira, os minérios, fundentes e o carvão vegetal são peneirados separadamente para a retirada do material fino com faixas granulométricas indesejáveis, obtendo, assim, melhoria no desempenho do alto-forno, uma vez que os espaços preenchidos pelos finos dificultam a passagem dos gases, reduzindo, assim, a produção do alto-forno.

A siderúrgica trabalha com seis tipos diferentes de minérios, e atualmente a empresa faz um blend (mistura) com dois desses minérios, levando em consideração a qualidade de cada um deles, a quantidade estocada nos pátios e o valor de mercado, entre outros fatores. A faixa granulométrica também é controlada, para melhorar a permeabilidade e distribuição da carga. Um fator importante usado pela empresa é a secagem de minérios, pois facilita a separação das impurezas no peneiramento.

O redutor utilizado no alto-forno é o carvão vegetal, que passa por um processo de peneiramento para separar o carvão a ser enfornado daqueles de faixa granulométrica baixa, que são destinados à moagem. Nesse processo, o carvão é transformado em um pó, que é injetado no altoforno. Esse pó injetado queima instantaneamente, tendo um tempo mais rápido de resposta, o que gera economia de carvão enfornado via topo pelos skips.

Por fim, os fundentes compõem a mistura que será carregada no alto-forno. Atualmente são utilizados pela empresa: manganês, dolomita, quartzo e calcário, responsáveis pela proteção da parte refratária do forno, contribuindo na composição química da escória. A empresa utiliza o método ternário para calcular a basicidade. Atualmente, o cálculo desse balanço de massa é feito através do método da tentativa e erro, levandose em consideração a experiência do operador.

\section{Modelagem do Problema}

Nesta seção é apresentado o modelo matemático baseado em Programação Linear por Metas, proposto para solucionar o Problema do Balanço de Massas, com melhor aproveitamento dos recursos disponíveis e redução do tempo gasto para calcular as quantidades que devem ser enfornadas.

\subsection{Parâmetros de entrada}

Sejam os seguintes parâmetros de entrada:

Produtos (i) - conjunto de produtos que podem ser alimentados pelo alto-forno;

Parâmetros ( $\mathbf{J})$ - conjunto de teores controlados no ferro-gusa e na escória;

$\mathbf{Q} \mathbf{u}_{\mathbf{i}}$ - quantidade disponível do produto i;

Prod - quantidade do material que deve ser

enfornada;

$\mathbf{c}_{\mathbf{i}}$ - custo do produto i;

$\mathbf{w d t}_{\mathbf{j}}$ - peso para o desvio da meta de qualidade do parâmetro j;

wdp - peso para o desvio da produção;

MetaQual $_{\mathbf{j}}$ - meta de qualidade para o parâmetro j;

$\mathbf{L I Q u a l}_{\mathbf{j}}$ - limite inferior para o parâmetro j; e

$\mathbf{L S Q u a l}_{\mathbf{j}}$ - limite superior para o parâmetro j;

tipo $_{i}=\left\{\begin{array}{l}1, \text { se o produto } i \text { for minério } \\ 0, \text { se o produto } i \text { for fundente }\end{array}\right.$

NumMin - número de tipos diferentes de minério que podem ser enfornados ao mesmo tempo.

\subsection{Variáveis de decisão}

Foram utilizadas as seguintes variáveis de decisão:

$\mathbf{x}_{\mathbf{i}}-$ quantidade que deverá ser enfornada do produto $\mathrm{i}$;

$\mathbf{D P M}_{\mathbf{j}}$ - desvio positivo da meta de qualidade do parâmetro de controle $\mathrm{j}$;

$\mathbf{D N M} \mathbf{M}_{\mathbf{j}}$ - desvio negativo da meta de qualidade do parâmetro de controle $\mathrm{j}$;

DPProd - desvio positivo da meta de produção;

DNProd - desvio negativo da meta de produção; e

$y_{i}=\left\{\begin{array}{l}1, \text { se o produto } i \text { for utilizado para formar a carga; } \\ 0, \text { c.c. }\end{array}\right.$ 
A função objetivo é:

$\min F O=\sum_{i \in \text { produtos }}\left(c_{i} x_{i}\right)+\sum_{j \in \text { parâmetros }}\left(w d t_{j}\left(D P M_{j}+D N M_{j}\right)\right)+w d p x(D P \operatorname{Pr} o d+D N \operatorname{Pr} o d)$

$$
\sum_{i} x_{i}+D P \operatorname{Pr} o d-D N \operatorname{Pr} o d=\operatorname{Pr} o d
$$

A função objetivo é composta por três partes: (i) que busca a minimização dos custos com a compra de material; (ii) que busca a minimização dos desvios das metas de qualidade; e (iii) que visa à minimização dos desvios das metas de produção.

\subsection{Restrições do problema}

enfornado:

Restrição de quantidade de material

\subsection{Restrições de controle da qualidade}

$$
\begin{array}{cc}
\sum_{i}\left(\text { teor }_{i j} x_{i}\right)+\text { DPM }_{j}-\text { DNM }_{j}=\text { MetaQual }_{j} \text { xQuant } & \forall j \in \text { parâmetros } \\
\sum_{i}\left(\text { teor }_{i j} x_{i}\right) \geq \text { LIQual }_{j} x \text { Quant } & \forall j \in \text { parâmetros } \\
\sum_{i}\left(\text { teor }_{i j} x_{i}\right) \geq \text { LSQual }_{j} x \text { Quant } & \forall j \in \text { parâmetros }
\end{array}
$$

usuário.

A equação 10 é responsável por medir os desvios da meta de qualidade dos parâmetros de controle.

Os conjuntos de equações 11 e 12 garantem que os limites, inferior e superior, respectivamente, não serão violados.

\subsection{Restrições para controlar o número de tipos} de minérios

$$
\begin{aligned}
& y_{i} \geq \frac{x_{i}}{Q u_{i}} \quad \forall i \in \text { produtos } \\
& \sum_{i \in \text { produtos } \text { tipo }_{i}=1} y_{i} \leq \text { NumMin }
\end{aligned}
$$

As equações 13 e 14 garantem que serão enfornados somente o número de tipos de minérios desejados pelo usuário. A primeira delas controla a variável $y_{i}$, forçando que ela seja igual a 1 se o minério do tipo $i$ for utilizado. A segunda garante que o somatório dos tipos de minério utilizados seja inferior ou igual ao número determinado pelo

\section{Dados de Entrada}

Para testar o modelo proposto foram utilizados os dados de entrada apresentados na Tabela 5.1. Nela são apresentados as quantidades e os teores de cada produto disponível para ser enfornado. 
Tabela 5.1 - Material disponível para serm enfornado

\begin{tabular}{|c|c|c|c|c|c|c|c|c|}
\hline & \multicolumn{7}{c|}{ Minério de ferro } \\
\hline & $\begin{array}{c}\text { Quant. } \\
\text { Disp. (kg) }\end{array}$ & \multicolumn{7}{c|}{ Teores (\%) } \\
\cline { 3 - 11 } & 15.000 & 63,70 & 2,05 & 1,79 & 0,09 & 0,61 & 0,13 & 1,91 \\
\hline Minério 01 & 20.000 & 67,52 & 1,57 & 0,79 & 0,05 & 0,08 & 0,06 & 1,21 \\
\hline Minério 02 & 12.000 & 64,16 & 3,69 & 1,92 & 0,08 & 0,06 & 0,11 & 1,93 \\
\hline Minério 03 & 17.250 & 63,80 & 1,77 & 1,77 & 0,13 & 0,64 & 0,11 & 1,93 \\
\hline Minério 04 & 21.500 & 66,10 & 1,13 & 1,48 & 0,06 & 0,36 & 0,06 & 2,55 \\
\hline Minério 05 & 12.500 & 63,33 & 3,86 & 1,99 & 0,07 & 0,41 & 0,09 & 3,28 \\
\hline Minério 06 & \multicolumn{7}{|c|}{ Fundentes } & \multicolumn{7}{c|}{ Teores (\%) } \\
\hline \\
\hline
\end{tabular}

As Tabelas 5.2 e 5.3 apresentam os parâmetros que precisam ser controlados no ferrogusa e na escória, respectivamente. Nelas são mostradas as metas e limites para cada um dos parâmetros de controle, bem como as quantidades de ferro-gusa e escória que deverão ser formados.

Tabela 5.2 - Requisitos do ferro-gusa

\begin{tabular}{|c|c|c|c|c|}
\hline \multicolumn{4}{|c|}{ Quantidade de ferro-gusa (kg) } & $\mathbf{5 0 2 0}$ \\
\hline & Fe & Si & P & Mn \\
\hline Lim. Máx. & & 0,80 & 0,10 & 0,75 \\
\hline Meta & 94,00 & 0,50 & 0,05 & 0,50 \\
\hline Lim. Min. & & 0,20 & & 0,25 \\
\hline
\end{tabular}

Tabela 5.3 - Requisitos da escória

\begin{tabular}{|c|c|c|c|c|c|}
\hline \multicolumn{5}{|c|}{ Quantidade de escória (kg) } & $\mathbf{3 8 0}$ \\
\hline & SiO2 & Al2O3 & CaO & MgO & IB3 \\
\hline $\begin{array}{l}\text { Lim. } \\
\text { Máx. }\end{array}$ & 47,00 & 17,00 & 29,00 & 15,00 & 0,94 \\
\hline Meta & 45,50 & 13,50 & 27,50 & 12,50 & 0,87 \\
\hline $\begin{array}{l}\text { Lim. } \\
\text { Min. }\end{array}$ & 44,00 & 10,00 & 26,00 & 10,00 & 0,80 \\
\hline
\end{tabular}

A Tabela 5.4 contém as demais informações necessárias, e nela são apresentados a quantidade de carvão vegetal que deverá ser enfornada e os percentuais de cinzas presentes no carvão e de óxido de cálcio nas cinzas, por fim a quantidade de tipos de minérios que podem ser enfornados juntos.

Tabela 5.4 - Demais informações

\begin{tabular}{|c|c|c|c|}
\hline \multicolumn{2}{|c|}{ Quantidade de carvão vegetal (kg) } & $\mathbf{2 4 0 0}$ \\
\hline $\begin{array}{c}\text { Cinzas no } \\
\text { carvão }\end{array}$ & $6,5 \%$ & CaO nas cinzas & $30,00 \%$ \\
\hline \multicolumn{3}{|c|}{$\begin{array}{c}\text { Número de minérios utilizados ao } \\
\text { mesmo tempo }\end{array}$} & $\mathbf{2}$ \\
\hline
\end{tabular}

\subsection{Resultados}

Nesta seção são apresentados os resultados encontrados pelo modelo matemático. $\mathrm{O}$ modelo foi executado em um computador AMD Athlon(tm) $3200+2.00 \mathrm{GHz}$ com $1 \mathrm{~GB}$ de RAM e aplicado a dados da empresa estudada.

$\mathrm{Na}$ Tabela 5.5 são exibidas as quantidades de cada um dos produtos que irão ser alimentados no alto-forno. 
Tabela 5.5 - Quantidades de produtos que deverão ser enfornados

\begin{tabular}{|c|c|c|c|c|c|c|c|c|}
\hline \multicolumn{9}{|c|}{ Minério de ferro } \\
\hline & \multirow{2}{*}{ Quant. (kg) } & \multicolumn{7}{|c|}{ Teores (\%) } \\
\hline & & $\mathbf{F e}$ & $\mathrm{SiO2}$ & Al2O3 & $\mathbf{P}$ & Mn & TiO2 & PPC \\
\hline Minério 01 & - & 64,07 & 4,60 & 1,58 & 0,09 & 0,04 & 0,13 & 1,91 \\
\hline Minério 02 & 6830 & 67,52 & 1,57 & 0,79 & 0,05 & 0,08 & 0,06 & 1,21 \\
\hline Minério 03 & - & 64,16 & 3,69 & 1,92 & 0,08 & 0,06 & 0,11 & 1,93 \\
\hline Minério 04 & 168 & 63,80 & 1,77 & 1,77 & 0,13 & 0,64 & 0,11 & 1,93 \\
\hline Minério 05 & - & 66,10 & 1,13 & 1,48 & 0,06 & 0,36 & 0,06 & 2,55 \\
\hline Minério 06 & - & 63,33 & 3,86 & 1,99 & 0,07 & 0,41 & 0,09 & 3,28 \\
\hline Global & 6998 & 67,43 & 1,57 & $\mathbf{0 , 8 1}$ & 0,05 & 0,09 & 0,06 & 1,23 \\
\hline \multicolumn{9}{|l|}{ fundentes } \\
\hline & \multirow{2}{*}{ Quant. (kg) } & \multicolumn{7}{|c|}{ Teores (\%) } \\
\hline & & $\mathbf{F e}$ & $\mathrm{SiO2}$ & Al2O3 & $\mathrm{CaO}$ & $\mathrm{MgO}$ & Mn & H2O \\
\hline Dolomita & 169 & 2,43 & 4,43 & & 28,69 & 26,12 & 0,07 & 4,00 \\
\hline Quartzo & 101 & & 95,30 & 4,66 & & & & 3,99 \\
\hline Calcário & 94 & 0,38 & 0,76 & & 57,06 & 3,87 & & 2,10 \\
\hline Manganês & 114 & 32,70 & 10,55 & 1,50 & & & 12,47 & 5,21 \\
\hline Global & 478 & 8,73 & 24,37 & 1,34 & 21,36 & 10,00 & 3,00 & 3,91 \\
\hline
\end{tabular}

A Tabela 5.6 apresenta os teores do ferro-gusa e da escória. Pode-se notar, nessa taabela, que todos os requisitos, quantitativos e qualitativos, foram atendidos.

Tabela 5.6 - Quantidade de teores encontrados no ferro-gusa e na escória

\begin{tabular}{|c|c|c|c|c|c|c|}
\hline \multirow{2}{*}{ Ferro-gusa } & Quant. (kg) & Fe & Si & P & Mn & \multirow{2}{*}{} \\
\cline { 2 - 6 } Escória & 5020 & 94,83 & 0,50 & 0,07 & 0,41 & \\
\hline \multirow{2}{*}{} & Quant. (kg) & $\mathbf{S i O 2}$ & $\mathbf{A l 2 O 3}$ & $\mathbf{C a O}$ & $\mathbf{M g O}$ & IB3 \\
\cline { 2 - 6 } & 380,00 & 45,50 & 14,98 & 27,00 & 12,57 & 0,87 \\
\hline
\end{tabular}

\section{Resultados e Discussão}

Este trabalho apresenta um modelo matemático capaz de solucionar o problema de balaço de massas apresentado em empresas de siderurgia.

A modelagem implementada do modelo foi desenvolvida utilizando o modelador e otimizador LINGO, versão 10.0, interfaceando com planilhas do Microsoft Excel. A utilização de planilhas facilita a operação do usuário, por the serem ferramentas conhecidas.

Pela análise dos resultados, pode-se concluir, também, que o modelo desenvolvido apresentou-se eficiente na resolução do problema. No estudo do caso analisado, o sistema encontrou a solução ótima para o problema rapidamente, atendendo a todas as suas restrições. A obtenção dos resultados pelo modelo demorou, em média, 10 seg, contribuindo com a drástica diminuição do tempo na tomada de decisão.

Outro aspecto que valida a importância deste trabalho foi a maior interação entre a aluna e empresas da região, possibilitando a utilização de técnicas aprendidas em sala de aula em problemas reais. Adicionalmente, contribui com a divulgação, no meio empresarial, de métodos de otimização na abordagem de um problema típico de empresas siderúrgicas.

É importante observar que, embora o trabalho tenha sido voltado para um estudo de caso, toda siderúrgica tem em seu processo problemas semelhantes. Assim, a solução a ser adotada no objeto de estudo pode ser utilizada ou adaptada para resolver problemas similares de outras empresas do ramo.

\section{Referências}

ALVES, José Maria do Carmo Bento. Um sistema para o planejamento de produção e vendas de 
uma empresa mineradora. 2007. 57 f. Dissertação (Mestrado em Engenharia Mineral) - Universidade Federal de Ouro Preto, Ouro Preto, 2007.

ARAUJO, Luiz Antonio de. Manual de siderurgia. São Paulo: Arte e Ciência, 1997. v. 1.

ARENALES, M.; ARMENTANO, V.; MORABITO, R.; YANASSE, H. Pesquisa operacional para cursos de engenharia. Rio de Janeiro: Campus, 2007.

BUENO, A. F.; OLIVEIRA, M. C. Goal programming (Programação Multiobjetivo). In: CORRAR, L. J.; THEÓPHILO, C. R. (Org.). Pesquisa operacional para decisão em contabilidade e administração. São Paulo: Atlas, 2004. Cap. 8, p. 394-438.

BREGALDA, P. F.; OLIVEIRA, A. A. F.; BORNSTEIN, C. T. Introdução à Programação Linear. 3. ed. Rio de Janeiro: Campus, 1988.

COSTA, F. P. Aplicações de técnicas de otimização a problemas de planejamento operacional de lavras em mina a céu aberto. 2005. 141 f. Dissertação (Mestrado em Engenharia Mineral) - Universidade Federal de Ouro Preto, Ouro Preto, MG, 2005.

MORAES, Edilaila Fernandes. Um modelo de Programação Matemática para Otimizar a Composição de Lotes de Minério de Ferro da Mina CAUÊ da CVRD. 2005. 78 f. Dissertação (Mestrado em Engenharia Mineral) - Universidade Federal de Ouro Preto, Ouro Preto, MG, 2005.

ROMERO, C. A general structure of achievement function for a goal programming model. European Journal of Operational Research, v. 153, p. 675686, 2004.

SENAI. Apostila de notas de aulas. Barão de Cocais, MG: Senai, 2008.

UNIVERSIDADE DE SÃO PAULO. Escola Politécnica. Disponível em: <http://publicacoes.pcc.usp.br/PDF/BT256.pdf>. Acesso em: 20 nov. 2009.
Artigo selecionado entre os 10 melhores do VII Encontro Mineiro de Engenharia de Produção - EMEPRO 2011. 